\title{
Entretien avec Yvonne Knibiehler
}

\section{Mathilde Dubesset et Françoise Thébaud}

\section{Q OpenEdition}

Journals

Édition électronique

URL : https://journals.openedition.org/clio/1707

DOI : 10.4000/clio. 1707

ISSN : 1777-5299

Éditeur

Belin

Édition imprimée

Date de publication : 1 avril 2005

Pagination : 247-268

ISBN : 2-85816-781-8

ISSN : $1252-7017$

Référence électronique

Mathilde Dubesset et Françoise Thébaud, «Entretien avec Yvonne Knibiehler », Clio. Histoire, femmes et sociétés [En ligne], 21 | 2005, mis en ligne le 01 juin 2007, consulté le 22 avril 2022. URL : http://

journals.openedition.org/clio/1707 ; DOI : https://doi.org/10.4000/clio.1707

Ce document a été généré automatiquement le 22 avril 2022

Tous droits réservés 


\title{
Entretien avec Yvonne Knibiehler
}

\author{
Mathilde Dubesset et Françoise Thébaud
}

1 La rubrique "Témoignage » a jusqu'ici privilégié l'interview d'actrices de l'histoire (résistante, cinéaste, théologienne, femme militaire, collectionneuse de tableaux...). Avec Yvonne Knibiehler, ce numéro inaugure l'interview d'historiennes et amorce une réflexion sur les carrières et les parcours intellectuels de celles qui se sont engagées en histoire des femmes et du genre et qui sont aussi actrices et témoins de leur temps. L'entretien s'est déroulé le 26 janvier 2005 dans une salle de l'IUFM de Marseille et a duré près de deux heures. Le texte a été relu par Yvonne Knibiehler.

2 Françoise Thébaud: Femmes, Familles, Filiations. Société et Histoire, livre d'hommage, présente votre itinéraire d'historienne engagée, itinéraire que nous souhaitons approfondir avec vous. Nous voudrions centrer l'interview sur le thème de la maternité, en développant trois points : d'abord, l'itinéraire d'une mère de famille du baby boom devenue universitaire; puis, le travail d'historienne de la maternité; enfin, Yvonne Knibiehler, militante de la maternité aujourd'hui.

3 Yvonne Knibiehler: Je précise : "militante de la maternité », cela ne signifie pas que j'invite toutes les femmes à être mères!

4 Mathilde Dubesset: Vous êtes née en 1922 et vous faites partie de la génération de femmes et d'hommes qui ont, en quelque sorte, fait le baby boom. Vous avez écrit que cette génération avait été celle du "consentement, de l'enfant accepté, non pas dans la docilité et la passivité mais par des femmes devenues citoyennes peu de temps avant et qui désiraient jouer un rôle actif dans la société ». Pourriez nous éclairer sur ce point en faisant un peu d' " ego-histoire "?

5 Y.K : Si la maternité tient tant de place dans mon travail et dans ma vie, c'est évidemment pour des raisons d'éducation. Mon père et ma mère appartenaient tous les deux à des familles nombreuses, et j'ai bien connu mes deux grands-mères qui étaient des femmes du XIXe siècle, pas du tout malheureuses. Quand on parle d'oppression des femmes, je préfère dire domination masculine parce que je ne puis admettre l'idée que mes deux grands-mères aient été des femmes opprimées. J'ai grandi parmi des ribambelles d'oncles, de tantes, et de cousins. C'était pour moi une joie. Mes parents n'ont eu que deux enfants. J'ai donc eu les deux modèles: le modèle de mes grandsparents, fécond, et le modèle, malthusien, de mes parents. Autre facteur important, 
dans les années 1938 et 1939, au lycée, on m’a appris que tout français conscient devait avoir au moins trois enfants. Il faut se remettre dans l'ambiance, on commençait à redouter la menace de guerre et on avait légitimement peur d'être écrasés par le grand nombre et la grande puissance des Allemands. Le déclin démographique de la France était déploré unanimement. Je crois que c'est Jean Zay (il faudrait vérifier, je n'en suis pas sûre) qui avait introduit dans les lycées, des enseignements de démographie invitant les jeunes à devenir parents d'au moins trois enfants. J'ai entendu cela comme un avertissement solennel et dramatique et j'ai décidé d'avoir trois enfants... (par patriotisme?) J'en conclus aujourd'hui que, grâce au système scolaire, on peut influencer les choix de vie des jeunes, avec le risque redoutable de les endoctriner (voir les jeunesses hitlériennes ou les gardes rouges de Mao).

$6 \quad$ F.T : Dans le cadre de quel cours, cette incitation était-elle faite ?

7 Y.K : en histoire et géographie, essentiellement. Les deux personnes qui nous ont fait ces cours étaient toutes deux des célibataires sans enfants, Mlle Foreville et Mlle Thouzellier, de belles personnalités avec lesquelles j'ai ensuite gardé des relations. Plus tard, je suis entrée à l'université et j'ai été une étudiante suffisamment brillante pour que le doyen de Montpellier, Augustin Fliche, médiéviste, m'invite à faire une thèse sur Bernard de Clairvaux. Je savais que si je m'engageais sur cette voie, c'était un choix de vie, je deviendrais une universitaire célibataire et savante. Cela me faisait peur, mais me tentait en même temps. Mon mari a réussi à faire pencher la balance dans l'autre sens. Nous étions avant la pilule, j'ai fait trois enfants en moins de cinq ans, au début de mon mariage, tous nés au Maroc (mon mari était ingénieur dans une mine). La maternité m'est apparue comme une révélation, je peux employer un terme aussi fort. J'ai pouponné avec enthousiasme, souvent fatiguée, mais si heureuse! J'ai ressenti que, pour une femme qui aimait cela (je ne prétends pas du tout que ce soit le cas de toutes les femmes), il y avait là un épanouissement, un accomplissement que rien n'égale. Je crois que c'est la motivation fondamentale qui m'a ensuite tournée vers l'histoire de la maternité. Après notre retour en France, j'ai enseigné dans les lycées pendant plus de quinze ans, parce que j'avais abandonné toute autre perspective, sans regret.

F.T : Pour revenir aux modèles familiaux et à ce que vous souhaitiez faire de votre vie, est-ce que dans votre famille il y avait autour de vous des femmes qui avaient une activité professionnelle extérieure? Est-ce que cela allait de soi ? Est-ce que votre mère travaillait?

Y.K : Non. Elle et ses sœurs n'ont eu qu'un ou deux enfants. Elles ne travaillaient pas à l'extérieur, ç'aurait été une honte pour les maris.

M.D : Et que faisait votre père?

11 Y.K : Mon père était fonctionnaire municipal à la mairie de Montpellier. Il a pris la direction de l'établissement des Bains et Lavoirs de la ville, pendant les années de l'entre-deux-guerres. Il gagnait bien sa vie, ma mère n'éprouvait pas le besoin matériel de travailler. Cela l'aurait intéressée pourtant, elle avait besoin d'indépendance, mais elle a renoncé sans peine.

12 F.T : Elle avait fait des études?

13 Y.K : Non. Mes parents n'avaient que le certificat d'études. Ils étaient tous les deux de familles nombreuses, issus de la petite bourgeoisie. Mes grands-parents maternels étaient commerçants et mon grand-père paternel était employé du chemin de fer PLM. Avec des familles nombreuses, ils ne pouvaient pas payer des études à leurs enfants. 
Mon père lui n'a voulu que deux enfants pour que ses enfants puissent faire des études, cela était clair pour lui.

F.T : Et vous, même en tant que fille, vous avez été poussée par vos parents pour faire des études?

Y.K : Oui. Mon père se voulait moderne dans ce domaine. Il a interdit que j'apprenne le piano, par exemple, parce que c'était une forme d'éducation qui détournait les jeunes filles des vraies études. J’ai été sevrée de musique. Pour le piano je ne le regrette pas, mais j'aurais voulu chanter, j'adorais le chant, cela reste un des regrets de ma vie. Mon père était décidé à ce que je fasse des études, et comme j'étais très bonne élève, dès l'école primaire, il m'a fait entendre qu'à ses yeux c'était cela qui comptait.

F.T : Le malthusianisme de vos parents - celui de "l'entre-deux-guerres ", est-il aussi un effet de la guerre ou de l'instabilité du monde?

Y.K : Cela je ne l'ai jamais entendu dire. Née en 1922, j'ai entendu les propos de ces hommes qui avaient fait la guerre. Tous les frères de mon père et de ma mère ont subi les quatre ans de guerre "au pire", si je puis dire : il y a eu un tué, il y a eu des blessés. J'ai vu des hommes accablés, démoralisés. Les enfants étaient comme une revanche, une consolation, une reconstruction de leur vie, nous avons été très choyés. Mais ils ne disaient pas qu'ils n'en voulaient que deux à cause de l'instabilité.

M.D : Dans la valorisation de la famille, de la maternité, est-ce que votre éducation religieuse a pu jouer un rôle?

Y.K : Je ne crois pas. Je suis devenue agnostique mais j'ai grandi dans le catholicisme. Il y avait des prêtres dans mes deux lignées. Je sais ce que je dois à ce type d'éducation. Je me rappelais, tout récemment, que la Toussaint et la fête des morts ( $1^{\text {er }}$ et 2 nov.), étaient célébrées en famille. Nous allions tous ensemble à la messe de la Toussaint et le lendemain tous ensemble - cela faisait du monde - au cimetière, d'une tombe à l'autre. La communion des saints, la communication entre les vivants et les morts m'ont été enseignés toute petite et je ressens encore à quel point c'était structurant, réconfortant. Mais ce n'est pas à l'Eglise qu'on m'a invitée à enfanter, jamais. Plus tard, le doyen Fliche, catholique pratiquant lui aussi, voulait que je fasse une thèse; il insistait: «On ne doit pas négliger les dons du ciel, vous pouvez faire une thèse sur Saint Bernard de Clairvaux, faites-la. » Pour lui c'était plus important que de faire des enfants, pour une fille comme moi.

F.T : Cela paraissait-il vraiment inconciliable à ce moment là de faire une thèse, de se marier et d'avoir des enfants?

Y.K : Absolument. M. Fliche, en me félicitant pour mon mariage a ajouté : «Vous êtes perdue pour l'histoire!» Mon père a tout fait pour me détourner du mariage, quel que soit le prétendant. Moi-même j'ai eu conscience de sacrifier ma carrière en me mariant (avec, pourtant, une petite réserve mentale : on verra plus tard)

F.T : Vous vous êtes mariée en quelle année?

Y.K : Je me suis mariée en 1949.

F.T : Après l'agrégation?

Y.K : Oui, j’ai été agrégée en 1945.

F.T: Toujours au sujet de votre formation, avez-vous lu Le Deuxième Sexe de Simone de Beauvoir au moment de sa parution en 1949 ? 
Y.K : Non. Je me suis mariée en juillet 1949, je suis partie au Maroc, j'ai eu mes enfants. Non. Je l'ai lu bien après.

M.D : Et quelle a été votre réaction à ce moment là ?

Y.K : Une espèce d'éblouissement. Elle a tout dit sur ce que, sans le formuler ou même le concevoir, nous ressentions. Nous, je veux dire les intellectuelles, les femmes qui avaient pu réfléchir sur la condition féminine, parce qu'elles avaient été contraintes à certains renoncements

F.T : Comment avez-vous fait, très concrètement, pour élever trois enfants et mener une carrière universitaire? Quand vous êtes entrée à l'Université d'Aix-en-Provence, au début des années 1960, étiez-vous une exception, comme universitaire et mère de famille nombreuse?

Y.K : Une exception, assurément. Vous savez c'est le hasard. Quand je suis entrée à l'université, le prof d'histoire contemporaine avait huit cents copies à corriger à l'examen (cela en raison du baby boom, de la démocratisation de l'enseignement supérieur, de l'arrivée des Pieds noirs). Ces messieurs aux abois cherchaient des gens pour les aider. Petite parenthèse : quand je suis arrivée à Aix j'ai demande une mise en disponibilité pour un an. Mon mari avait quitté les mines pour l'énergie atomique, il avait travaillé à Marcoule (et nous habitions Avignon), puis il avait été envoyé à Cadarache, et nous sommes venus habiter Aix, mais il n'y avait aucun poste pour moi à Aix, d'où ma demande de congé.

F.T : En quelle année?

Y.K : En 1962. Je ne suis pas une ménagère. Quand j'ai eu fait le déménagement et rangé toute la maison, je suis allée voir à l'université ce qui se passait. Je suis tombée sur M. Jean-Rémi Palanque, antiquiste brillant, qui, à Montpellier, m'avait connue comme étudiante. Il m'a reconnue, vingt ans après, je n'en revenais pas. Et il m'a dit : «Si vous avez le désir de venir enseigner à l'université, vous serez la très bienvenue ». J'ai pensé : « Pourquoi pas, c'est peut-être le moment ». Mes enfants, avaient douze, onze et huit ans, ils pouvaient se débrouiller. L'année suivante, j'ai eu un poste à Aix, au lycée Cézanne. Mais, comme agrégée, j'étais vouée aux classes de terminales, première et seconde, j'avais l'impression de rabâcher, j'en avais assez. Puisque j'avais été médiéviste, je suis allé voir Georges Duby, qui brillait de tous ses feux. Il m'a reçue très courtoisement, mais sans me laisser aucun espoir : il avait fait des promesses à ses meilleurs disciples aixois. Par contre, Pierre Guiral, en histoire contemporaine, avait besoin d'aide : il m'a offert des vacations, que j'ai acceptées.

F.T : Vous avez abandonnéle lycée?

Y.K : Non. La première année j'étais encore au lycée, et en plus je donnais quatre heures par semaine en « propédeutique ». C'était lourd, mais bon, je l'ai fait. Guiral m'a alors conseillé de déposer un sujet de thèse, et m'a suggéré une biographie de Mignet, qui avait été le grand homme d'Aix au XIX siècle. C'est ce que j'ai fait. J'espérais trouver beaucoup de sources à Aix : il y en avait, mais l'essentiel était à Paris, et j'ai dû assumer de nombreux déplacements.

36 F.T : À la recherche, en ce moment, des femmes docteures ès lettres du XXe siècle, je découvre l'existence de plusieurs types de thèses (thèse d'Université, thèse d'Etat, puis bientôt thèse de troisième cycle). La vôtre s'appelait comment ? Je crois que vous parlez de " petite thèse »...

Y.K : Je crois l'avoir déposée comme thèse de troisième cycle, ce qui devait me permettre d'être titularisée à l'université, mais non pas de devenir professeur. Pourtant 
j'ai acquis rapidement la conviction que la thèse d'état était à ma portée Guiral répétait partout qu'une thèse, c'est l'œuvre d'une vie, qu'il faut que le conjoint soit d'accord, sinon ça brise les ménages... C'était le discours qu'il tenait, lui et tous les autres! Quand Guiral m'a présentée au doyen Bernard Guyon, un littéraire, père de neuf enfants, ce dernier a ri : "Madame, on n'entreprend pas une thèse à quarante ans, surtout si on a des enfants ! » Je me suis crue capable d'un tel exploit : capable de faire une thèse d'État sans ruiner ma vie de femme, de mère, et en gardant des loisirs. J'avais raison. La thèse d'Université, c'était tout autre chose: elle permettait de reconnaître le travail de chercheurs qui n'avaient pas le cursus universitaire, par exemple des instituteurs, des inspecteurs du travail, ou des étrangers. Elle n'ouvrait aucun droit, elle était honorifique. Alors que les hommes mettaient dix ans pour faire leur thèse d'Etat, j'ai mis neuf ans. Cela ne m'a pas paru aussi difficile qu'on le prétendait.

F.T : En quelle année avez-vous soutenu votre thèse?

Y.K : En novembre 1970. Je dois dire que la même année mon père et ma mère sont morts à deux mois d'intervalle, en mars et mai 1970. Mon père est mort à Montpellier. Juste après j'ai pris ma mère chez moi, j'ai découvert qu'elle avait un cancer, dissimulé jusque là ; je l'ai soignée comme un bébé, mais sans espoir. Comme pour toute mère et toute fille, nos rapports avaient connu des hauts et des bas; elle adorait mes enfants, qui nous avaient tout à fait rapprochées. Sa fin a été bouleversante. Venant à la suite de ces traumatismes, ma soutenance ne m'apparaissait plus guère que comme une formalité.

\section{F.T : Quels étaient les membres du jury?}

Y.K : Le président était le doyen Jacques Godechot de Toulouse. Le rapporteur, Pierre Guiral, bien sûr. Il avait été déconcerté par mon travail: il attendait une étude d'histoire politique sur Mignet auxiliaire de Thiers ; or Mignet avait voulu être d'abord un historien, comme ses contemporains Thierry ou Michelet. J'avais cherché à comprendre ce que cela signifiait, et j'avais fait une thèse d'histoire de l'histoire, sur le conseil d'Alphonse Dupront, merveilleux éveilleur d'idées, que j'avais eu comme professeur à Montpellier, et que j'avais consulté au début de ma recherche. Le troisième membre du jury était Maurice Agulhon, qui aimait beaucoup ma thèse, ce qui m'a donné de l'assurance. Le quatrième était un professeur de Grenoble dont le nom ne veut pas me revenir.

F.T : Donc quatre hommes au jury.

Y.K: Quatre hommes. Il n'y avait pas de femmes à ce moment là. J'ai été la première dans la section d'histoire d'Aix à soutenir et à devenir professeur.

F.T : Vous étiez assistante?

Y.K : J'étais « maître-assistant ». J'ai été assistante un ou deux ans, pas plus, et maîtreassistant après.

M.D : Il y a eu, dans votre parcours, une part de hasard...

Y.K : C'est évident. J'ai eu une chance, au bon moment et je l'ai saisie.

M.D : Vous êtes rapidement devenue professeur finalement?

Y.K : J'ai soutenu en 1970, j'ai été professeur en 1972, sur la chaire de Maurice Agulhon quand il est parti à l'Institut. 

temps, vos enfants, vous les aviez fait avant d'y entrer. Est-ce que les enfants ne posaient pas problème pour une femme professeur de l'enseignement secondaire? dans un lycée de garçons. Mes congés de maternité étaient mal vus: trois mois de congés payés. Un mufle m'a laissé entendre que j'aurais dû me débrouiller pour faire mes enfants pendant les vacances. aujourd'hui ? Où situez-vous les évolutions ou les réticences? point de vue de la vie privée je ne sais pas. Dans les années 1950-1960 on trouvait facilement à se faire aider. J'ai eu des bonnes à temps complet ou des femmes de ménage, quatre heures tous les matins, pour assurer le travail matériel; je me suis toujours bien entendue avec elles (à une seule exception près). J'arrivais pour me mettre à table à midi avec les enfants. Nous étions mieux aidées, maintenant c'est la croix et la bannière pour trouver des femmes de service. et votre cheminement intellectuel, à vous intéresser à l'histoire des femmes? Et tout particulièrement à l'histoire de la maternité ? Je rappelle que l'Histoire des mères du Moyen Âge à nos jours est parue en 1980. L'introduction de l'ouvrage, coécrit avec Catherine Fouquet, s'intitule: "Les mères ont-elles une histoire?", et vous vous y définissez toutes les deux comme mères et historiennes. Nos questions portent à la fois sur la part de subjectivité de l'historienne dans l'écriture de l'histoire, sur le pourquoi faire l'histoire des femmes, des mères, sur vos découvertes.

Y.K : Pour ce qui est de l'histoire des femmes, c'est dans le sillage de Mai 68 et le début des années 1970. Vous savez la part que j'ai prise dans l'histoire du CEFUP (Centre d'études féminines de l'Université de Provence). L'histoire des femmes, c'est dans ce groupe que je l'ai conçue et construite. Qu'est-ce que l'on pouvait faire en histoire des femmes? Je me le suis demandée à ce moment là et j'ai trouvé des réponses. J'ai demandé à créer une unité d'histoire des femmes.

Y.K : Ceux qui me connaissaient bien, comme Joutard, Agulhon, avant qu'il parte, Guiral, Vovelle, l'antiquiste Paul Albert Février..., ceux-là comprenaient parfaitement ce que j'avais envie de faire, et m'ont soutenue. Mais d'autres trouvaient cela ridicule, notamment un préhistorien et deux disciples de Duby. Le préhistorien m'a dit en rigolant : « Pourquoi pas l'histoire des hommes ? » À quoi j'ai répondu : « On y viendra bientôt. » L'un des disciples de Duby (cela m'a été rapporté) a eu des plaisanteries d'une grossièreté allant jusqu'à l'obscénité. De ce côté j'ai eu une belle revanche, puisque quelques temps après Duby a donné à l'histoire des femmes un éclatante consécration! J'ai tenu bon grâce au CEFUP. Des historiens y sont venus, Marcel Bernos par exemple. Avec lui et Eliane Richard nous avons pu fonder une unité, qui ne s'est pas appelée histoire des femmes, pour ne choquer personne, mais histoire de la famille. Nous y traitions de la famille, honnêtement, mais en appuyant du côté des femmes. Ainsi nous avons pendant trois années de suite fait travailler les étudiants sur la jeune fille, " personne et personnage ». Les obstacles qui barraient la route à l'histoire des femmes ont été bientôt emportés par les flots de la grande contestation soixante huitarde.

Clio. Femmes, Genre, Histoire, 21 | 2005 

intéressée à l'histoire des femmes, je me suis dit: "Qu'est-ce qu'elle a de spécifique? " J'ai bientôt perçu que la différence la plus indéniable se situait dans le corps, la sexualité et la maternité ; grâce aux conseils de Jacques Léonard, j'ai trouvé un gisement de sources d'une richesse époustouflante: le discours des médecins. Mon premier grand article, paru dans les Annales en 1976, avait pour titre «Les médecins et la nature féminine au temps du code civil. Il a fait sensation. J'éprouvais moi-même le besoin très subjectif d'explorer cette différence. Comment est-elle perçue ? Comment pèse-t-elle sur la vie des femmes et sur leur histoire? Il y avait la sexualité, sujet important, mais pour moi moins important que la maternité. La maternité était encore pensée comme hors du temps, comme liée à la nature, donc éternelle et universelle. En réalisant L'histoire des mères, je voulais montrer que, justement, la maternité avait une histoire, qu'elle était un objet d'histoire. Une fois engagée, j'ai aperçu des développements tellement immenses que même maintenant, je n'en vois pas les limites. On peut repenser toute l'histoire humaine à travers l'histoire de la maternité.

\section{F.T : Et votre collaboration avec Catherine Marand-Fouquet?}

Y.K : Catherine venait au séminaire de Michel Vovelle, elle était moderniste, elle connaissait mieux que moi les sociétés préindustrielles, et j'ai pensé que nous pouvions partager le travail. Je la voyais aussi excellente chercheuse. Autre chose m'a touchée. Elle avait deux enfants, et un jour, dans nos conversations, elle a laissé échapper qu'elle mourait d'envie d'en avoir un troisième. Ça a fait tilt. Après la naissance de nos deux premiers enfants, fille et garçon, mon mari trouvait que c'était très bien, ça suffisait. C'est moi qui ai voulu le troisième, un peu à cause de l'enseignement de démographie que j'avais reçu, et aussi en raison du plaisir que j'avais éprouvé à pouponner. J'ai eu ce troisième enfant. Quand j'ai entendu ce même désir chez Catherine, je me suis sentie proche d'elle, et je lui ai proposé une collaboration. Elle a accepté avec joie. Nous nous sommes partagé le travail, la répartition chronologique donnant à chacune le maximum de liberté. Tout s'est bien passé. Nous avons eu pourtant une cruelle déconvenue : Elisabeth Badinter a publié trois mois avant nous L'amour en plus, et nous a coupé l'herbe sous le pas.

61 F.T : Ce livre était-il une commande de l'éditeur ou de votre initiative?

62 Y.K : La commande venait de Daniel Roche. Il était très lié à un couple qui se lançait dans l'édition de livres illustrés et qui cherchait des idées nouvelles. D'ailleurs, à ce moment-là, on recevait des démarcheurs, qui venaient nous dire : "Qu'est-ce que vous faites comme cours Madame? Est-ce que vous voudriez publier sur votre cours?». Quand Daniel Roche m'a posé la question, je le connaissais à peine, mais on s'est entendu tout de suite. Ses amis éditeurs ont pris l'idée à cœur, et ils ont réalisé un très bel ouvrage, qui mettait notre texte en valeur : nous étions comblées.

63 M.D : Vous l'avez vous-même dit, l'ouvrage d'Elisabeth Badinter est sorti quelques mois avant le vôtre. Quelle a été votre réaction à ce livre?

Y.K : J'étais désolée parce qu'évidemment il nous nuisait d'une manière catastrophique et en plus il contenait des erreurs d'interprétation qui ont été corrigées ensuite. Au départ ce n'était pas un bon livre. Quand j'ai eu à parler sur mon livre et le sien, j'ai mis en évidence les défauts du sien : elle ne me l'a jamais pardonné. 
d'une éducation, d'une inculcation. C'est vrai bien sûr. Ce qui est humain passe toujours par la culture, on le savait avant elle. Mais son analyse est simpliste. Que penser du désir d'enfant chez les mammifères que nous sommes? Certes il ne faut pas maudire les femmes qui n'«aiment " pas leurs enfants, mais le plus urgent est de se demander pourquoi, et de trouver des moyens pour améliorer cette relation. 
de mes maternités, mais aussi de mon penchant pour le travail intellectuel. J'ai pris plaisir à faire ma thèse parce que j'y trouvais l'occasion de réfléchir sur ma discipline, l'histoire. Les passerelles se sont ensuite construites toutes seules entre mon travail d'historienne et ma vie de mère. Pourquoi les aurais-je maintenus séparées?

81 F.T : Le questionnement " histoire des femmes » était difficilement pensable dans l'Université au moment où vous faisiez votre thèse. Michelle Perrot raconte, dans son essai d'ego-histoire, avoir évoqué avec Ernest Labrousse en 1949 le désir d'entamer des recherches sur le féminisme. Labrousse avait souri et lui avait proposé de travailler sur les coalitions ouvrières. Et vous, dans les années 1960, avez-vous évoqué avec Guiral la possibilité de faire une recherche sur l'histoire des femmes?

Y.K : Pas avec Guiral. Mais dans ma thèse la question s'est posée. Mignet a eu une longue liaison avec la princesse Belgiojoso qui était une personnalité de tout premier plan: grande patriote, elle militait ardemment au service de l'unité italienne. Son activité politique choquait la bourgeoisie du temps de Louis-Philippe; la presse l'a traînée plus bas que terre, traitée de folle et de putain. J'ai pris fait et cause pour elle. J'ai écrit un article qui a paru dans une revue italienne et j'ai continué à parler d'elle, à l'occasion. Elle m'a aidée à prendre conscience de mon féminisme. Je pense que j'ai longtemps été féministe sans le savoir. Le seul fait que j'ai été la seule dans ma ribambelle de cousins à faire des études supérieures m'a donné l'idée de ma propre valeur et de la façon dont je voulais conduire ma vie. La princesse affichait un indifférence souveraine face aux calomnies, mais, à travers elle, j'ai vu quels moyens étaient utilisés pour humilier les femmes et les écarter de la vie publique

F.T : Et vous l'avez écrit dans votre thèse?

Y.K : J'ai montré toute l'estime que méritait cette femme.

F.T : Le jury a-t-il relevé?

Y.K: Non, le jury n'a pas fait attention. Des choses qui me paraissaient à moi essentielles, le jury ne les voyait pas. Autre exemple, j'ai posé clairement une question épistémologique : «Qu'est-ce que l'histoire ? Pourquoi faisons-nous de l'histoire ?». J'ai essayé de voir pourquoi Mignet en a fait, et je trouvais léger de ne pas me demander pourquoi moi j'en faisais, pourquoi les membres de mon jury en faisaient. Aucun de ces messieurs n'a daigné entendre. Pourquoi font-ils de l'histoire? Parce que c'est bien de faire de l'histoire. Pourquoi est-ce bien? Parce qu'ils en font.

F.T : Pour revenir au CEFUP, le terme "études féminines » a-t-il été discuté ? D'autres centres se sont appelés d'études féministes. Y a-t-il eu débat ? Que signifiait ce choix? Y.K : Oui, cela a été discuté. La décision a été prise à la majorité. Nous pensions que le terme "féministes" risquait d'écarter un certain nombre de gens qui pouvait être intéressés par nos travaux. Il y avait alors dans le mot "féministe" une agressivité que nous ne voulions pas afficher.

F.T : Entre "études féminines » et "études sur les femmes», y a-t-il eu aussi débat? Quelle différence faisiez-vous?

90 Y.K : Personnellement, je n'y ai jamais réfléchi. Dans le groupe, on a pensé «études féminines", et cela voulait dire à la fois sur les femmes et par les femmes, probablement.

91 F.T : Bien que le CEFUP soit quand même un groupe mixte.

92 Y.K : Oui, il y avait des hommes. 

question maternelle ou de la maternité. Rappelons d'abord votre itinéraire de recherche. Après l'Histoire des mères en 1980, vous explorez, soit avec Catherine Fouquet, soit avec d'autres, soit seule, d'autres territoires du féminin : les rapports entre femmes et médecins, le temps des jeunes filles, les métiers féminins comme assistante sociale ou infirmière. Vous initiez aussi des recherches sur les femmes au temps des colonies. À la fin des années 1990, vous revenez à la maternité, en publiant en 1997 La révolution maternelle, qui porte comme sous-titre: Femmes, Maternité, Citoyenneté depuis 1945. Avant et après cet ouvrage qui achève en quelque sorte votre histoire des mères et de la maternité, vous inaugurez ce que nous appelons, et vous allez nous dire si vous êtes d'accord, une sorte d'engagement civique sur les questions de maternité. Vous considérez-vous aujourd'hui comme une militante sur les questions de maternité? en sciences sociales ayant pour thème la maternité. Sur ce chantier, la relève ne me paraît pas assurée. Les féministes de la seconde vague n'ont accepté la maternité que comme épanouissement narcissique du moi féminin, comme une affaire purement privée, expression de la liberté individuelle de chacune; donc elles n'en parlent plus. Mais moins on en parle et plus elle pèse. Les effets pervers de ce silence se révèlent dangereux. D'abord, la recherche est abandonnée aux médecins qui voient surtout des corps souffrants, et aux psy qui voient des relations affectives noyées dans les ténèbres de l'inconscient ; pour les uns et les autres, la maternité reste très loin de la vie sociale, elle est sans rapport avec les œuvres de l'intellect. Ensuite, la plupart des femmes souhaitent enfanter, et l'idylle maman/bébé constitue pour elles une composante majeure de l'épanouissement du moi féminin; or cette idylle est souvent gâchée par des conditions socio-économiques qui contraignent la salariée à choisir entre ses tâches maternelles et ses tâches professionnelles; elle n'a aucun moyen de forcer le père à s'investir, et d'ailleurs beaucoup de mères élèvent seules leurs enfants. Enfin, la liberté de ne pas enfanter a mis en évidence le désir d'enfant. La «libre maternité » s'est bientôt affirmée comme un "droit à l'enfant ", entraînant un essor imprévu et quasi incontrôlable de l'adoption d'une part et des procréations assistées d'autre part, avec les perspectives vertigineuses que l'on sait. La procréation restera l'arme absolue de la domination masculine tant que les féministes n'auront pas fait l'effort de penser et de construire la maternité comme un élément original, spécifique de la liberté et de l'identité féminines. La liberté de ne pas être mère étant acquise, reste à conquérir la liberté d'être mère, sans se perdre.

F.T : Parlons de la Société d'Histoire de la naissance, née en 2000, que vous présidez. Pouvez-vous revenir sur sa création, ses objectifs et les actions qu'elle mène?

Y.K : Je ne suis pour rien dans sa création. Elle a été créée par un médecin obstétricien, le docteur Paul Cesbron, chef de service de la maternité de Creil. Il aime l'histoire et il a eu envie de développer un séminaire. Il n'est pas enseignant, je pense que cela lui manque, il a besoin de dialogue. Il connaissait bien Marie-France Morel. Il a été un militant passionné au moment de la loi Veil en faveur de l'avortement, et il est encore aujourd'hui président, je crois, de l'Association nationale des centres d'interruption de grossesse (ANCIC). Quand il a lu l'Histoire des mères et ensuite La révolution maternelle, il a trouvé que je conciliais (c'est lui qui me l'a dit) le féminisme avec son métier, qui était de faire naître des enfants. Il adore ce métier. Il m’a demandé de présider la société. J’ai accepté, au moins pour les trois premières années, j'ai fait tout ce que j'ai pu pour

Clio. Femmes, Genre, Histoire, 21 | 2005 
assister aux délibérations et pour jouer un rôle dynamique. Un premier colloque (Châteauroux, 2002) avait traité de l'accouchement sans douleur (voir ci après un compte rendu d'ouvrage), un second (Nantes 2004) a traité de l'histoire des sages femmes. J'ai rassemblé les témoignages de 70 à 80 sages-femmes dont j'espère tirer un recueil. Outre des sages femmes, viennent surtout aux réunions des médecins (gynécologues, obstétriciens, anesthésistes), et aussi, épisodiquement, des psy, des sociologues, des anthropologues et des historien(ne)s... civilisation et que l'on ne peut plus renier. C'est l'autre face de l'humanisme. L'humanisme a été défini comme universel parce les humanistes étaient des hommes, en écrasante majorité; pour eux, l'universel c'était le masculin. Qu'est-ce que l'humanisme? C'est une doctrine qui prend pour fin la personne humaine et son épanouissement. Mais la personne humaine est sexuée: ce qui permet l'épanouissement d'une personne de sexe masculin peut ne pas suffire, ou ne pas 
convenir à l'épanouissement d'une personne de sexe féminin. La différence des sexes n'a pas d'autre raison d'être originelle que la reproduction, et tant que la reproduction se fera par les corps, elle conditionnera les femmes autrement que les hommes. On peut effectivement réduire ces différences (toute ma vie prouve que j'ai refusé d'être réduite à la condition de mère qui accouche et pouponne). Mais il faut permettre à une femme de s'épanouir dans la maternité si elle le souhaite, au moins pour quelques années. Une rationalisation de la fécondité est désormais acquise : peu de gens veulent des familles nombreuses mais peu de gens renoncent à avoir des enfants, ils en veulent entre un et quatre (quand on a fait trois filles on veut un garçon (ou l'inverse) donc on a quatre enfants). Pourquoi pas? Reconnaître la différence des sexes dans la procréation, en tenir compte, est-ce revenir au naturalisme ? Non, absolument pas. Il s'agit au contraire de dépasser à tout jamais la naturalisation patriarcale, d'en sortir par le haut.

F.T : Quelles mesures préconisez-vous pour permettre aux femmes qui souhaitent être mères de vivre mieux leur maternité aujourd'hui?

Y.K : D'abord que les femmes, les mères, se pensent enfin comme des citoyennes et qu'elles prennent en main leur propre destin sans trop attendre des hommes, ni de l'Etat. En elles, un sujet mère vient renforcer le sujet femme L'Etat c'est elles aussi. La maternité cessera d'être aliénante pour les mères quand une volonté politique puissante les animera. Le sujet mère devrait s'appliquer à réveiller le sujet père chez les citoyens de sexe masculin. La citoyenneté a pris jusqu'à présent une forme abstraite, ignorant la différence des sexes, c'est à dire, en fait, ignorant les femmes et les enfants ; elle n'a pas donné à la "parentalité » des moyens d'expression suffisants. Concrètement, il faut chercher des aménagements collectifs, une organisation sociale qui facilite la vie des parents. Je trouve dommage que les femmes n'y réfléchissent pas toutes ensemble, qu'elles pensent toujours la maternité comme une affaire purement privée. Il y a en France des associations pour tout. Il y a des associations de parents : pourquoi sont-elles si discrètes? Il faudrait inventer des partages, des relais, des institutions... Une autre chose me gêne. Beaucoup de mères recourent à des assistantes maternelles; celles-ci reçoivent une formation qualifiante qui justifie un salaire décent, mais la mère, elle, ne reçoit aucune formation qualifiante: pourra-t-elle dialoguer d'égale à égale avec son assistante?

M.D : Il y a, dans les politiques publiques, des choses qui peuvent être incitatives. Des pays nordiques ont instauré un congé de paternité obligatoire. En France, le récent congé de quinze jours, pour les pères, est quand même un signe intéressant. Qu'en dites-vous?

108 Y.K : C'est très bien. Je crois que le congé de paternité a obtenu un succès inespéré : plus de $40 \%$ des pères en ont profité. Mais quand un homme a du temps libre il n'a pas toujours envie de le consacrer à sa progéniture. Il y aurait peut-être toute une éducation à entreprendre.

109 M.D : On a l'impression, à vous entendre et à vous lire, que vous mettez un peu en alerte la société devant une sorte de péril qui menacerait la famille. Un livre est paru il y a quelque temps, d'Evelyne Sullerot qui est de votre génération, et qui posait aussi cette question. Votre engagement actuel est-il lié à cette idée d'une sorte de désagrégation de la famille ?

110 Y.K : Les jeunes croient ma génération suspecte de nostalgie, donc un peu radoteuse. Soupçon injuste, inacceptable. Est-ce être réactionnaire de souhaiter que des parents et leurs enfants soient heureux ensemble? Ce n'est pas de la sacro sainte famille que je me soucie : elle a connu des formes diverses dans le passé, elle continue à se transformer, c'est normal. Le problème à résoudre aujourd'hui - et il n'est pas facile, c'est vrai - c'est 
la conciliation de l'individualisme avec des engagements qui permettent l'éducation des enfants dans les meilleures conditions. L'individualisme est certes un progrès, il faut le confirmer, le consacrer (les femmes ont trop souffert d'un déficit d'individualisme), mais il faut aussi apprendre à l'intégrer dans la vie sociale! Or les engagements de caractère juridique ne suffisent pas, les liens affectifs comptent beaucoup. Un enfant a besoin que ses parents l'aiment, même s'ils le font élever par d'autres. On est là au cœur des difficultés actuelles.

111 F.T : Votre objectif, notamment à travers la Société d'Histoire de la naissance, est-il de faire en sorte que les femmes, celles qui le souhaitent du moins, puissent vivre de la meilleure façon possible à la fois l'accouchement, mais aussi l'éducation de leurs enfants? Qu'il y ait une invention collective autour de ce thème?

112 Y.K : Oui, c'est cela. Prenons conscience qu'il y a là quelque chose d'essentiel. D'abord pour les femmes : il faut qu'elles s'en rendent compte elles-mêmes, pour l'enfant, mais aussi pour les hommes, indirectement.

113 F.T : Vous l'avez dit, la maternité serait trop considérée comme une affaire privée aujourd'hui?

114 Y.K : Oui, on n'a pas pris suffisamment en compte sa dimension sociale.

115 M.D : Pensez-vous que le féminisme n'a pas pris à bras le corps cette question?

116 Y.K : C'est bien mon regret. Cela s'explique historiquement très bien en ce sens que le féminisme a redémarré après le baby-boom, à un moment où la maternité était non pas imposée légalement aux femmes, mais présentée comme une vocation prioritaire. Du coup, les féministes, à commencer par Simone de Beauvoir, ont regardé la maternité comme une aliénation. Elles n'avaient pas tort à ce moment là. J'ai souvent entendu dire moi-même que la mère se doit à ses enfants, son métier passant après. C'était imposer aux femmes d'abolir leur personnalité, de renoncer à leurs aptitudes. Quand le féminisme de la seconde vague s'est affirmé, il a repris ce thème de l'aliénation; il a dit aussi aux femmes que la maternité était un choix privé, qu'elles étaient libres de se donner ce plaisir mais sans importuner les autres. Sois mère et tais toi !

117 M.D : Il y a eu tout de même un courant de valorisation de la maternité, avec Annie Leclerc et tous les développements sur la féminitude...

118 Y.K : Annie Leclerc exaltait le corps en termes narcissiques. Elle célébrait avec un lyrisme outrancier la joie de la grossesse («le ventre», dirait M. Iacub), pour établir une supériorité de la féminitude. Il y a aussi des gens qui prétendent qu'on jouit en accouchant: ce sont souvent des gynéco qui font état de leur expérience professionnelle. C'est peut-être vrai pour quelques femmes : combien? une sur mille? On peut, certes opposer la maternité jouissance à la maternité aliénation (je le fais moimême), mais pas en ces termes là. Revenons à Simone de Beauvoir, son message est beaucoup plus complexe et nuancé qu'on ne le croit. La maternité, dit-elle en substance, n'accomplit la femme que si elle est voulue et assumée en pleine et entière responsabilité. L'enfant n'est pas une justification toute faite, c'est un engagement auquel on peut se consacrer, mais sous certaines conditions : une femme ne devrait donner la vie que si la vie a un sens, et si elle peut elle-même déterminer ce sens en participant activement à la vie de la cité. Son rapport à l'enfant s'inscrit dans les rapports sociaux : il doit être assumé par la collectivité. Ces propos contenaient en germe une idée encore peu explorée, même aujourd'hui : la relation entre la maternité et la citoyenneté. La citoyenneté avait été accordée aux femmes en 1944 sans tenir compte de leur fonction maternelle (réelle ou potentielle), de même qu'elle avait été 
accordée aux hommes en 1848, sans tenir compte de leur fonction paternelle (réelle ou potentielle). Les responsabilités parentales, et surtout maternelles se sont alourdies à la fin des années 1960, par l'effet des lois Neuwirth (1967) et Veil (1975), qui confèrent aux femmes un habeas corpus spécifique Une femme "libérée " qui impose la vie à un enfant " désiré » a non seulement le droit, mais plus encore le devoir de participer, avec compétence et vigilance, à l'élaboration du monde où cet enfant grandira. Tels sont, je crois, au seuil du XXIe siècle, les nouveaux fondements, socio- culturels de la maternité

119 M.D : Dans la conclusion de La révolution maternelle, vous appelez à vivre la maternité comme une composante de la citoyenneté. Est-ce que vous pouvez préciser?

120 Y.K : La première forme de notre démocratie a pensé le citoyen comme un être abstrait, indépendamment de son sexe, de sa classe, de sa race. Or, je pense que nous devons entrer dans un second âge de la démocratie où le citoyen sera un être concret, c'est à dire où il pourra introduire dans ses engagements politiques certains éléments fondamentaux de sa personnalité, en particulier son sexe, et surtout son engagement dans la procréation. Quand un homme et une femme s'engagent dans la procréation, leur citoyenneté est différente. Ils sont responsables pour eux-mêmes mais responsables aussi de l'avenir pour l'enfant ou les enfants qu'ils mettent au monde.

121 M.D : Est-ce que cela voudrait dire que des gens qui n'ont pas d'enfants ne pourraient pas se sentir aussi responsables vis-à-vis des jeunes générations dont ils peuvent avoir la charge?

Y.K : Je pense qu'une génération est globalement responsable de la suivante en ce sens que les gens qui n'ont pas d'enfants s'occupent très souvent des enfants des autres, les enseignants, les médecins, les policiers, les juges. D'ailleurs tout adulte peut servir de modèle identificatoire pour les jeunes, parfois même à son insu; il doit en prendre conscience et assumer cette responsabilité

F.T : Nous voudrions, pour finir, évoquer avec vous les grands débats du moment qui divisent la société et aussi les féministes : la prostitution, l'homoparentalité, les procréations médicalement assistées. Ainsi, la prostitution est-elle, pour vous, un métier comme un autre?

124 Y.K : Le grand problème est de savoir si la prostitution est forcée ou si elle est libre. Tout est là. Ceux et celles qui, comme Elisabeth Badinter ou Marcela Iacub, défendent la possibilité de la prostitution comme métier, disent qu'une femme a le droit de louer son corps contre de l'argent. Pourquoi pas? Jusque-là, je peux les suivre. Mais massivement, dans notre société actuelle, la prostitution est forcée. C'est une exploitation et pas seulement des femmes... Telle qu'elle je ne peux pas l'admettre, c'est évident. J'ai connu des étudiantes qui faisaient des passes de temps en temps pour arrondir leurs fins de mois. Qu'est-ce que l'on peut dire à cela? On ne peut pas prétendre que les femmes sont libres et leur disputer la liberté sur ce point précis, ce serait du moralisme. Je ne vais pas condamner la prostitution d'un point de vue moral, mais je trouve que c'est toujours dangereux de la légitimer sous prétexte qu'elle peut être libre, parce que dans $99 \%$ des cas elle ne l'est pas. Il faut absolument être très prudent dans ce domaine.

M.D : Autre sujet d'actualité, l'homoparentalité: après le PACS, la revendication de l'accès au mariage, pour les homosexuels, s'exprime fortement ainsi que le droit d'élever des enfants et de faire famille. Qu'en pensez-vous?

Y.K. J'ai lu le livre d'Anne Cadoret Des enfants comme les autres, ainsi que d'autres travaux. J'ai été récemment invitée par l'Association des parents gays et lesbiens à faire 
une conférence-débat. J'ai rencontré là des gens extrêmement réfléchis, scrupuleux, sympathiques. Entre des géniteurs irresponsables, qui s'enivrent tous les soirs, et ces gens là qui réfléchissent des années avant de se procurer un enfant, je préfère les seconds. On peut dire, évidemment, qu'il est souhaitable qu'un enfant soit élevé par un homme et une femme. J'ai connu des mères lesbiennes, qui donnent deux parrains à chacun des enfants pour qu'ils aient deux figures masculines identificatoires, qu'ils ne soient pas réduits à des modèles féminins. Il paraît que dans les pays où l'on a un recul suffisant, en Hollande par exemple, il y a des enfants de parents homosexuels qui ont maintenant 16-18 ans, et ce sont des enfants comme les autres. Des psychanalystes répondent qu'on le verra vraiment quand ils auront eux-mêmes des enfants. Il faut attendre leur désir d'enfant et leur engagement dans la procréation pour être sûrs qu'ils seront des parents comme les autres. Ceci dit, si les parents hétérosexuels étaient tous des modèles à imiter, on pourrait jeter la pierre aux parents homosexuels. Ce n'est pas le cas. Je n'ai pas des positions tranchées dans ce domaine, je suis en alerte..

F.T : Et sur la question des mères porteuses, interdites en France?

Y.K : C'est un thème à propos duquel j'ai évolué, en lisant Marcela Iacub. Au début, évidemment, cela me choquait profondément. Et puis progressivement je me suis dit mais après tout pourquoi pas? Autrefois il $\mathrm{y}$ avait des nourrices qui donnaient leur lait, elles élevaient au sein le bébé d'une autre et personne ne s'en portait plus mal. Alors, pourquoi ne pourrait-on pas louer l'utérus? Si c'était interdit partout, s'il y avait un consensus partout, je serais plutôt d'accord avec le consensus hostile. Mais du moment que les gens hésitent et qu'il y a possibilité en passant la frontière de trouver une mère porteuse, alors je trouve que la loi française ne tient plus, elle perd des justifications. Parlant de la procréation, j'aimerais aussi qu'il y ait un contrepoids. Il faudrait, que au cours de leur l'éducation, garçons et filles apprennent qu'on peut avoir une vie très féconde et très utile sans enfanter soi-même. Cette possibilité d'être un citoyen utile sans procréer n'est pas suffisamment indiquée, on n'en parle pas avec les enfants. La passion d'enfanter à tout prix est presque aussi choquante que les procédés les plus extravagants de procréation médicalement assistée. En tant que féministe, je vois que comme toujours les femmes en paient le prix: ce sont elles qui subissent les traitements éprouvants; on peut à bon droit se demander si ces procédés méritent d'être défendus " à tout prix », d'autant qu'ils sont extrêmement coûteux.

F.T : Comment voyez-vous la société d'aujourd'hui. Sommes-nous dans une situation de backlash où les droits des femmes seraient menacés? Faut-il plutôt dire qu'il y a de grandes différences entres femmes et de grandes différences entre sociétés sur cette question-là ?

Y.K : Je suis plutôt d'accord avec le second point. En tant qu'universitaire, je vois se développer les études sur les femmes, même si elles ne sont pas officiellement reconnues. Vous ne demandez pas forcément l'autorisation pour tenir des séminaires ou des colloques sur les femmes et on peut les faire passer sous d'autres titres. La Société d'Histoire de la naissance, par exemple, travaille essentiellement sur ce qui intéresse les femmes, elle est peuplée de sages-femmes, de femmes-médecins et d'historiennes. J'ai l'impression d'une progression régulière de la réflexion sur les femmes, des savoirs sur les femmes. Je ne pourrai donc pas admettre le mot "backlash". Mais, il me semble qu'il y a des différences selon les milieux. J'en reviendrai à la dimension sociale de la maternité. Si chacune ne s'enfermait pas dans sa propre expérience, si elle avait le souci de rencontrer d'autres mères, de discuter de maternité, peut-être que les choses évolueraient plus facilement. Il y aurait des échanges entre les 
femmes des différents milieux. Cela pourrait se faire par l'intermédiaire de puéricultrices, d'institutrices d'écoles maternelles, de directrices de crèches, de travailleuses sociales... Ces personnes voient des femmes de presque tous les milieux et elles peuvent agir en étant discrètes. Il faudrait une socialisation de la parentalité. Quand je dis "socialisation", je ne l'entends pas au sens des soviets! Non pas une socialisation imposée par les pouvoirs mais celle qu'organiseraient les parents, entre eux, en toute liberté.

131 F.T : Vous êtes très sensible à la question des responsabilités citoyennes des individus, ainsi qu'à la transmission d'une génération à l'autre. Pourriez-vous revenir sur la question de la transmission? Que voulez-vous transmettre aujourd'hui? Qu'est-ce que nos sociétés ne savent pas transmettre? Qu'est-ce qu'il serait urgent de faire pour engager la collectivité sur ces questions de maternité et de parentalité?

132 Y.K : Il me paraît central de transmettre les valeurs démocratiques. La démocratie, est elle même une pédagogie. Elle existe pour que les gens prennent en mains leur propre destin. Transmettre cette conception de la démocratie me paraît capital. Une femme peut la transmettre déjà dans sa vie quotidienne, l'enseigner à ses enfants : c'est une responsabilité de mère. La différence des sexes doit être enseignée aux jeunes très tôt, dés qu'ils en prennent conscience, vers trois ans ; je sais que cela se fait, ici ou là, au gré des instituteurs; ce devrait être systématique. Les petits doivent comprendre que la différence des sexes est nécessaire à la procréation, mais nous ne sommes pas des bêtes, nous sommes des humains, nous avons une conscience ; à chacun, chacune de choisir (ou non) de devenir parents. Si l'on veut des enfants, on prend la responsabilité de tout ce qui suivra: les conditions de la naissance, les soins, l'éducation. À mesure que les enfants grandissent, cette responsabilité doit être de mieux en mieux explicitée. S'engager dans la maternité, la paternité, la parentalité, c'est rester vigilant, s'occuper des enfants d'une manière politique, élaborer des réformes s'il y a lieu. Non pas qu'il faille rêver d'un monde parfait, mais parce que le but de la démocratie doit être de vivre ensemble le moins mal possible. Tout cela, une femme peut le transmettre, c'est l'une des obligations de la maternité. Une mère transmet beaucoup, consciemment et inconsciemment. Elle peut et doit maitriser ce qu'elle veut transmettre consciemment. Quand je parle de maternité, je ne la dissocie pas de la paternité. La maternité est un élément de la parentalité, mot nouveau qui s'impose de plus en plus ; il réunit le père et la mère et les oblige à penser ensemble. L'homme pense la maternité, la femme pense la paternité : excellent exercice qui devrait les aider à progresser l'un et l'autre, et à faire progresser la société. Un effort devrait être fait également du coté du système scolaire : chargé de l'instruction des jeunes, il ne peut pas se désintéresser de leur éducation, notamment sexuelle; il peut et doit «les aider à grandir » comme disait Françoise Dolto. Par exemple en marquant des seuils (entrée à l'école primaire, au collège, au lycée), qui remplaceraient les anciens "rites de passage ». Mais ceci est une autre histoire...

F.T et M.D : Merci à vous. 